\title{
Performance Evaluation of Micropattern Representation on Gabor Features for Face Recognition
}

\author{
Sanqiang Zhao \\ QRL, National ICT Australia \\ IIIS, Griffith University \\ Brisbane, Queensland, Australia \\ sam.zhao@nicta.com.au \\ s.zhao@griffith.edu.au
}

\author{
Yongsheng Gao \\ School of Engineering, Griffith University \\ QRL, National ICT Australia \\ Brisbane, Queensland, Australia \\ yongsheng.gao@griffith.edu.au \\ yongsheng.gao@nicta.com.au
}

\author{
Baochang Zhang \\ School of Automation Science and \\ Electrical Engineering \\ Beihang University \\ Beijing, China \\ bczhang@buaa.edu.cn
}

\begin{abstract}
Face recognition using micropattern representation has recently received much attention in the computer vision and pattern recognition community. Previous researches demonstrated that micropattern representation based on Gabor features achieves better performance than its direct usage on gray-level images. This paper conducts a comparative performance evaluation of micropattern representations on four forms of Gabor features for face recognition. Three evaluation rules are proposed and observed for a fair comparison. To reduce the high feature dimensionality problem, uniform quantization is used to partition the spatial histograms. The experimental results reveal that: 1) micropattern representation based on Gabor magnitude features outperforms the other three representations, and the performances of the other three are comparable; and 2) micropattern representation based on the combination of Gabor magnitude and phase features performs the best.
\end{abstract}

Keywords-Face recognition; performance evaluation; micropattern representation; Gabor feature

\section{INTRODUCTION}

Recently, face recognition using micropattern representation has received much attention in the computer vision and pattern recognition community [1-10]. One such micropattern representation, the Local Binary Pattern (LBP) [1], was first used for texture classification [2], but later extended to background modeling [3], face detection [4], expression analysis [5] and face recognition [6]. The micropattern representation has the advantages of high discrimination capacity, invariance against monotonic grayscale changes and computational efficiency. There have been a lot of researches devoted to improving the performance of LBP. For example, Zhao et al. [7] proposed a Sobel-LBP to enhance the performance of LBP using the Sobel operator. Zhang et al. [8] proposed a Local Derivative Pattern (LDP) to encode high-order multi-directional micropatterns for face recognition. To further increase the discrimination ability, the micropattern representation has been extended from gray-level images to Gabor magnitude features [9] and Gabor phase features [10]. In both situations, Gabor feature based micropattern representation significantly outperformed gray-level image based micropattern representation. However, an objective evaluation and fair comparison of micropattern representations on different Gabor features has not been reported in the literature.

In this paper, we conduct a comparative performance evaluation of micropattern representations on four forms of Gabor features (real part, imaginary part, magnitude and phase) for face recognition. Three evaluation rules are proposed and observed to conduct an objective and fair comparison. The three evaluation rules can exclude other interferential factors and thus ensure that our experiments deal exclusively with Gabor feature-level comparison of micropattern representations. To reduce the high feature dimensionality problem, the uniform quantization method is used to partition the spatial histograms of Gabor feature based micropatterns. The experimental results reveal that: 1) micropattern representation based on Gabor magnitude features outperforms the other three representations, and the performances of the other three are comparable; and 2) micropattern representation based on the combination of Gabor magnitude and phase features performs the best.

In the following, Section II introduces four forms of Gabor feature based micropattern representations. Section III proposes three rules observed for fair performance evaluation. Section IV conducts comparative experiments on the publicly available FERET database [11]. The last section concludes the paper with some discussions.

\section{MiCROPATTERN REPRESENTATION ON FOUR FORMS OF GABOR FEATURES}

Gabor wavelets have been widely used in image processing and pattern recognition. The 2D Gabor kernel functions used for feature extraction are

$$
\psi_{j}(\mathbf{z})=\frac{k_{j}^{2}}{\sigma^{2}} \exp \left(-\frac{k_{j}^{2} z^{2}}{2 \sigma^{2}}\right)\left[\exp \left(i \mathbf{k}_{\mathrm{j}} \mathbf{z}\right)-\exp \left(-\frac{\sigma^{2}}{2}\right)\right],
$$

where

$$
\mathbf{k}_{\mathbf{j}}=\left(\begin{array}{c}
k_{j x} \\
k_{j y}
\end{array}\right)=\left(\begin{array}{c}
k_{v} \cos \varphi_{\mu} \\
k_{v} \sin \varphi_{\mu}
\end{array}\right), k_{v}=2^{-\frac{v+2}{2}} \pi, \varphi_{\mu}=\mu \frac{\pi}{8} .
$$

The index $j=\mu+8 v$ covers a discrete set of five different frequencies $v=0, \cdots, 4$ and eight orientations $\mu=0, \cdots, 7$. The width of the Gaussian is controlled by the parameter 
$\sigma=2 \pi$. The Gabor features $G_{j}(\mathbf{z})$ of a given image $I(\mathbf{z})$ are defined as its convolution with 40 Gabor kernels: $G_{j}(\mathbf{z})=I(\mathbf{z}) * \psi_{j}(\mathbf{z})$.

\section{A. Gabor Feature Based LBP}

Let $G_{j}^{\mathrm{Re}}, G_{j}^{\mathrm{Im}}, G_{j}^{\mathrm{Ma}}$ and $G_{j}^{\mathrm{ph}}$ denote the Gabor real part, imaginary part, magnitude and phase features of an image, respectively, where $j=0, \cdots, 39$. For each position $G_{j, c}$ on a Gabor feature image, the Gabor feature based LBP $\left(G_{-} L B P\right)$ is calculated by thresholding the $P$ equally spaced neighbor positions $G_{j, p, R}(p=0, \cdots, P-1)$ on a circle of radius $R$ with the central position $G_{j, c}$ and concatenating the results binomially with factor $2^{p}$ :

$$
\begin{aligned}
& G_{-} L B P_{j, P, R}^{\mathrm{Re}}=\left\{\sum_{p=0}^{P-1} s\left(G_{j, p, R}^{\mathrm{Re}}-G_{j, c}^{\mathrm{Re}}\right) \times 2^{p}\right\}, \\
& G_{-} L B P_{j, P, R}^{\mathrm{Im}}=\left\{\sum_{p=0}^{P-1} s\left(G_{j, p, R}^{\mathrm{Im}}-G_{j, c}^{\mathrm{Im}}\right) \times 2^{p}\right\}, \\
& G_{-} L B P_{j, P, R}^{\mathrm{Ma}}=\left\{\sum_{p=0}^{P-1} s\left(G_{j, p, R}^{\mathrm{Ma}}-G_{j, c}^{\mathrm{Ma}}\right) \times 2^{p}\right\}, \\
& G_{-} L B P_{j, P, R}^{\mathrm{Ph}}=\left\{\sum_{p=0}^{P-1} s\left(G_{j, p, R}^{\mathrm{Ph}}-G_{j, c}^{\mathrm{Ph}}\right) \times 2^{p}\right\},
\end{aligned}
$$

where the thresholding function $s(x)$ is defined as

$$
s(x)=\left\{\begin{array}{ll}
1, & x \geq 0 \\
0, & x<0
\end{array} .\right.
$$

Fig. 1 illustrates visualized examples of $G_{-} L B P^{\mathrm{Re}}$, $G_{-} L B P^{\mathrm{Im}}, G_{-} L B P^{\mathrm{Ma}}$ and $G_{-} L B P^{\mathrm{Ph}}$ representations for a face image from the FERET database.

\section{B. Uniform Quantization of G_LBP Spatial Histograms}

The histogram of G_LBP micropatterns contains important discriminative information. To preserve structural information, a Spatial Histogram $(S H)$ which concatenates the histograms of several non-overlapping rectangular subregions is employed to represent a face:

$$
\begin{array}{r}
S H^{\mathrm{Re}}(I)=\left\{H_{G_{-} L B P_{j}^{\mathrm{Pe}}}\left(R_{i}\right) \mid i=1, \cdots, N ; j=0, \cdots, 39\right\}, \\
S H^{\mathrm{Im}}(I)=\left\{H_{G_{-} L B P_{j}^{\mathrm{Pm}}}\left(R_{i}\right) \mid i=1, \cdots, N ; j=0, \cdots, 39\right\}, \\
S H^{\mathrm{Ma}}(I)=\left\{H_{G_{-} L B P_{j}^{\mathrm{Ma}}}\left(R_{i}\right) \mid i=1, \cdots, N ; j=0, \cdots, 39\right\}, \\
S H^{\mathrm{Ph}}(I)=\left\{H_{G_{-} L B P_{j}^{\mathrm{Ph}}}\left(R_{i}\right) \mid i=1, \cdots, N ; j=0, \cdots, 39\right\},
\end{array}
$$

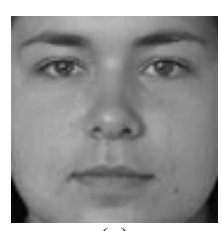

(a)

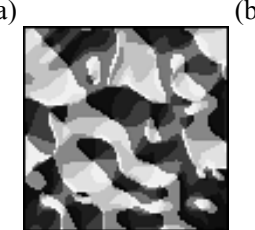

(d)

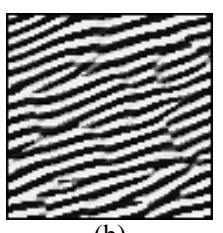

(b)

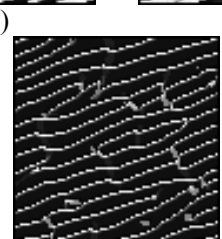

(e)
Figure 1. Visualization of $G_{L} L B P \mathrm{~s}(j=11)$. (a) Original image. (b) $G_{-} L B P^{\mathrm{Re}}$. (c) $G_{-} L B P^{\overline{\mathrm{Im}}}$. (d) $G_{-} L B P^{\mathrm{Ma}}$. (e) $G_{-} L B P^{\mathrm{Ph}}$.

where $H_{G_{-} L B P_{j}}\left(R_{i}\right)$ denotes the histogram of $G_{-} L B P$ micropatterns extracted from the $i^{\text {th }}$ subregion $R_{i}(i=1, \cdots, N)$.

It should be noted that the micropatterns extracted from four forms of Gabor features induce a high dimensionality of the histogram vector (the feature length of each form of $G_{L} L B P$ is 40 times as long as that of image LBP). To solve this problem, the uniform quantization method is utilized to partition the subregion histogram with equal intervals. If each subregion histogram is quantized into $B$ histogram bins, these $B$ bins with equal size $256 / B$ can be represented as: $[0, \cdots, 256 / B-1] \quad, \quad[256 / B, \cdots, 2 \times 256 / B-1] \quad, \quad \cdots \quad$, $\left[(B-1) \times{ }^{256} / B, \cdots, 255\right]$. The selection of an optimal number of histogram bins is a compromise between recognition performance and speed.

There are many similarity measures for histogram matching. Considering its simplicity and efficiency, the histogram intersection measure is used to compare two histograms in this paper.

\section{EVALUATION RULES}

To ensure an objective and fair comparison, the $G_{-} L B P$ operator implemented and experimented in this paper is a very basic version $(P=8, R=1)$ without any performance enhancement techniques such as subregion weighting or more complicated histogram measurement.

We also vary the subregion size and number of histogram bins to conduct the comparison under different parameters, and we observe the following three evaluation rules:

1) The Equal-Feature-Length rule, i.e., the histogram features of different micropattern representations must be of the same length;

2) The Same-Encoding-Operation rule, i.e., the micropatterns on different Gabor features must be encoded through the same thresholding function (using the central position) followed by a circular bit-wise concatenation; and

3) The Same-Encoding-Parameter rule, i.e., the micropattern representations on different Gabor features must be compared using the same set of 
parameters, such as subregion size and number of histogram bins.

\section{EVALUATION RESUlts}

The comparative experiments are conducted on the publicly available FERET database [11] from four probe sets (FB, FC, Dup I and Dup II) against the gallery set FA. The four probe sets contain frontal face images with expression, illumination and aging variations. All the images are normalized and cropped to $88 \times 88$ pixels based on the positions of two eyes.

\section{A. Comparisons among Four Forms of Gabor Features}

Fig. 2 illustrates the top-one recognition accuracies of four forms of Gabor feature based LBPs ( $G_{-} L B P^{\mathrm{Re}}$, $G_{-} L B P^{\mathrm{Im}}, G_{-} L B P^{\mathrm{Ma}}$ and $G_{-} L B P^{\mathrm{Ph}}$ ) performed on four FERET probe sets with the same subregion size of $11 \times 11$ pixels. The average recognition accuracy on four probe sets is also listed here for comparison. Three different numbers of histogram bins $(8,16$ and 32$)$ in each subregion are tested. It can be seen from the figure that an increasing number of histogram bins improves the recognition rates of $G_{-} L B P^{\mathrm{Re}}$, $G_{-} L B P^{\mathrm{Im}}$ and $G_{-} L B P^{\mathrm{Ph}}$, but $G_{-} L B P^{\mathrm{Ma}}$ has comparatively stable performance with different histogram bins. We further fix the number of histogram bins in each subregion to 16 , and examine the influence of different subregion sizes on the recognition accuracy. The experimental results in Fig. 3 demonstrate that $G_{-} L B P^{\mathrm{Re}}$ and $G_{-} L B P^{\mathrm{Im}}$ are very sensitive to subregion partition, in contrast to $G_{-} L B P^{\mathrm{Ma}}$ and $G_{-} L B P^{\mathrm{Ph}}$, whose performances are comparatively stable against this variation. Table I lists the numerical results of the best performances of each micropattern representation with corresponding optimal parameters. Although each representation reaches its upper-bound performance at different set of parameters, the performance of $G_{-} L B P^{\mathrm{Ma}}$ is much better than those of the other three representations, especially on the FC probe set. The overall performances of $G_{-} L B P^{\mathrm{Re}}, G_{-} L B P^{\mathrm{Im}}$ and $G_{-} L B P^{\mathrm{Ph}}$ are comparable. These results reveal the robustness of Gabor magnitudes against expression variation, illumination change and local distortion, and indirectly explain the reason why Gabor magnitudes have been widely used in many face recognition systems.

\section{B. Comparisons between Combined Gabor Features}

We also conduct comparative experiments on combined Gabor feature based LBPs, i.e., LBP from concatenated Gabor real part and imaginary part features $\left(G_{-} L B P^{\mathrm{Re}+\mathrm{Im}}\right)$ and LBP from concatenated Gabor magnitude and phase features ( $\left.G_{-} L B P^{\mathrm{Ma}+\mathrm{Ph}}\right)$. Fig. 4 displays the top-one recognition accuracies with the same subregion size of $11 \times 11$ pixels and different histogram bins $(8,16$ and 32$)$ in each subregion. It can be inferred from the figure that $G_{-} L B P^{\mathrm{Ma}+\mathrm{Ph}}$ performs consistently better than $G_{-} L B P^{\mathrm{Re}+\mathrm{Im}}$.
$G_{-} L B P^{\mathrm{Ma}+\mathrm{Ph}}$ also outperforms separate use of either Gabor magnitude ( $G_{-} L B P^{\mathrm{Ma}}$ ) or phase features $\left(G_{-} L B P^{\mathrm{Ph}}\right)$, which indicates that Gabor magnitude and phase features contain different discriminative information and their micropattern representations may compensate each other. However, the performance of $G_{-} L B P^{\mathrm{Re}+\mathrm{Im}}$ is similar to or slightly better than that of separate use of Gabor real part $\left(G_{-} L B P^{\mathrm{Re}}\right)$ or imaginary part features ( $\left.G_{-} L B P^{\mathrm{Im}}\right)$, which reveals that the discriminative information contained in the micropattern representations of Gabor real part and imaginary part features is overlapping. Therefore, when using Gabor real part and imaginary part features for micropattern representation, it may be adequate to use any one form of features.

\section{CONCLUSIONS}

This paper conducts a comparative performance evaluation of micropattern representations based on four forms of Gabor features for face recognition. The experimental results indicate that Gabor magnitude feature based micropattern representation outperforms the other three representations. The performances of Gabor real part, imaginary part and phase feature based micropattern representations are comparable. The experimental results also reveal that the discriminative ability of micropattern representation based on Gabor magnitude features can be improved by combining with Gabor phase features, indicating Gabor magnitude and phase features are good compensation for each other. However, micropattern representation based on concatenated Gabor real part and imaginary part features does not perform much better than individual feature based micropattern representations.

TABLE I. NUMERICAL RESULTS OF THE BEST RECOGNITION ACCURACIES OF $G$ _LBPS WITH CORRESPONDING PARAMETERS

\begin{tabular}{|c|c|c|c|c|}
\hline $\begin{array}{c}\text { Recognition } \\
\text { Rate }(\%) \\
\end{array}$ & $\begin{array}{c}\operatorname{Re}(11 \times 11, \\
32 \text { bins })\end{array}$ & $\begin{array}{c}\text { Im }(11 \times 11, \\
16 \text { bins })\end{array}$ & $\begin{array}{c}\text { Ma }(4 \times 4, \\
16 \text { bins })\end{array}$ & $\begin{array}{c}\text { Ph }(8 \times 8, \\
16 \text { bins })\end{array}$ \\
\hline FB & 87.28 & 86.95 & 90.96 & 88.70 \\
\hline FC & 80.41 & 76.80 & 88.14 & 79.38 \\
\hline Dup I & 53.32 & 53.74 & 58.17 & 54.43 \\
\hline Dup II & 49.57 & 50.43 & 55.56 & 53.42 \\
\hline Average & 67.65 & 66.98 & 73.21 & 68.98 \\
\hline
\end{tabular}

\section{REFERENCES}

[1] T. Ojala, M. Pietikäinen, and D. Harwood, "A Comparative Study of Texture Measures with Classification Based on Feature Distributions," Pattern Recognition, vol. 29, pp. 5159, 1996.

[2] T. Ojala, M. Pietikäinen, and T. Mäenpää, "Multiresolution Gray-Scale and Rotation Invariant Texture Classification with Local Binary Patterns," IEEE Transactions on Pattern Analysis and Machine Intelligence, vol. 24, pp. 971-987, 2002 .

[3] M. Heikkilä and M. Pietikäinen, "A Texture-Based Method for Modeling the Background and Detecting Moving Objects," IEEE Transactions on Pattern Analysis and Machine Intelligence, vol. 28, pp. 657-662, 2006.

[4] A. Hadid, M. Pietikäinen, and T. Ahonen, "A Discriminative Feature Space for Detecting and Recognizing Faces," 
Proceedings of the IEEE Computer Society Conference on Computer Vision and Pattern Recognition, pp. 797-804, 2004.

[5] G. Zhao and M. Pietikäinen, "Dynamic Texture Recognition Using Local Binary Patterns with an Application to Facial Expressions," IEEE Transactions on Pattern Analysis and Machine Intelligence, vol. 29, pp. 915-928, 2007.

[6] T. Ahonen, A. Hadid, and M. Pietikäinen, "Face Description with Local Binary Patterns: Application to Face Recognition," IEEE Transactions on Pattern Analysis and Machine Intelligence, vol. 28, pp. 2037-2041, 2006.

[7] S. Zhao, Y. Gao, and B. Zhang, "Sobel-LBP," Proceedings of the IEEE International Conference on Image Processing, pp. 2144-2147, 2008.

[8] B. Zhang, Y. Gao, S. Zhao, and J. Liu, "Local Derivative Pattern Versus Local Binary Pattern: Face Recognition with
High-Order Local Pattern Descriptor," IEEE Transactions on Image Processing, vol. 19, pp. 533-544, 2010.

[9] W. Zhang, S. Shan, W. Gao, X. Chen, and H. Zhang, "Local Gabor Binary Pattern Histogram Sequence (LGBPHS): A Novel Non-Statistical Model for Face Representation and Recognition," Proceedings of the IEEE International Conference on Computer Vision, pp. 786-791, 2005.

[10] B. Zhang, S. Shan, X. Chen, and W. Gao, "Histogram of Gabor Phase Patterns (HGPP): A Novel Object Representation Approach for Face Recognition," IEEE Transactions on Image Processing, vol. 16, pp. 57-68, 2007.

[11] P.J. Phillips, H. Moon, S.A. Rizvi, and P.J. Rauss, "The FERET Evaluation Methodology for Face-Recognition Algorithms," IEEE Transactions on Pattern Analysis and Machine Intelligence, vol. 22, pp. 1090-1104, 2000.

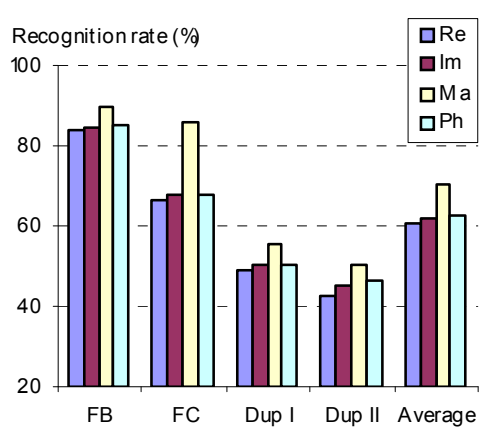

(a) 8 histogram bins

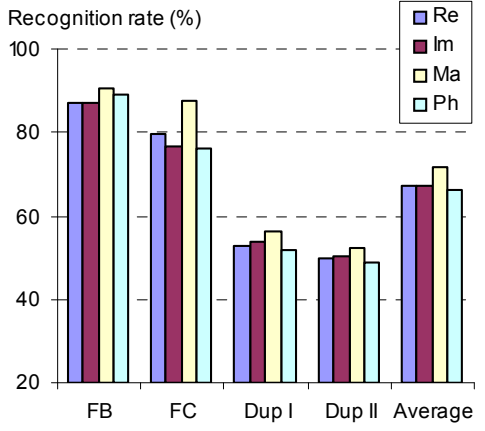

(b) 16 histogram bins

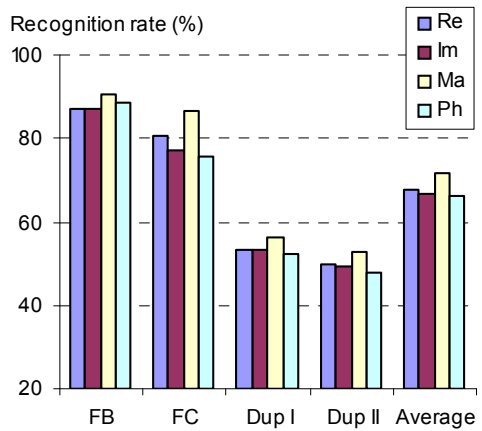

(c) 32 histogram bins

Figure 2. Comparative top-one recognition rates of $G_{-} L B P \mathrm{~s}$ with $11 \times 11$-sized subregions.

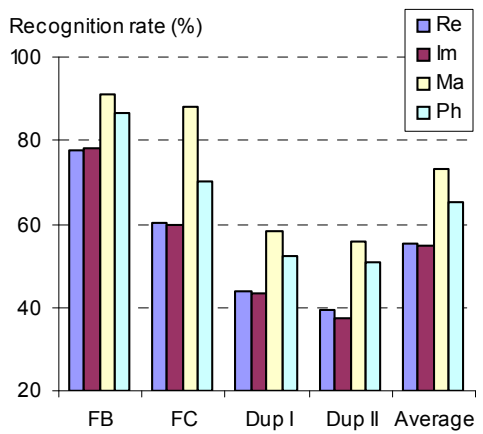

(a) $4 \times 4$-sized subregions

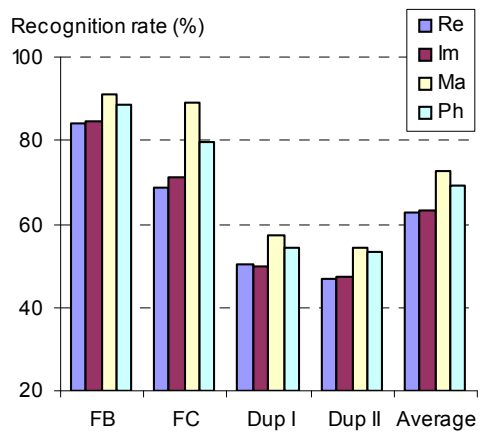

(b) $8 \times 8$-sized subregions

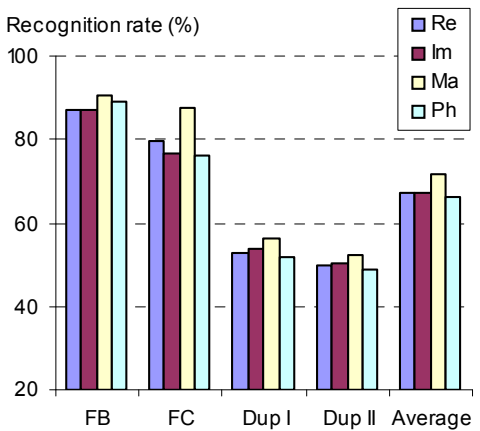

(c) $11 \times 11$-sized subregions

Figure 3. Comparative top-one recognition rates of $G_{-} L B P$ s with 16 histogram bins.

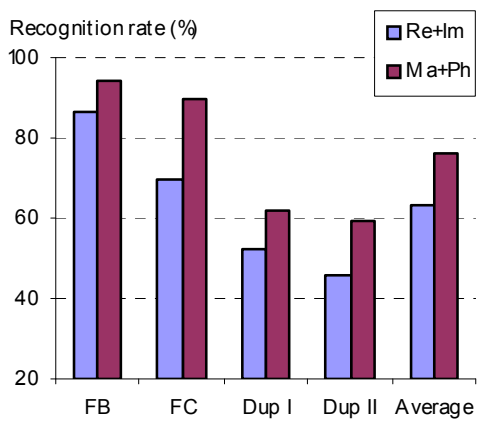

(a) 8 histogram bins

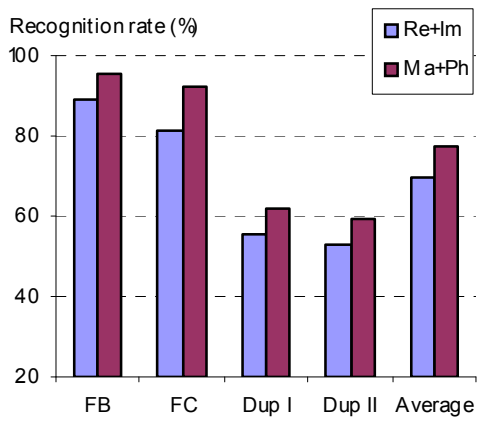

(b) 16 histogram bins

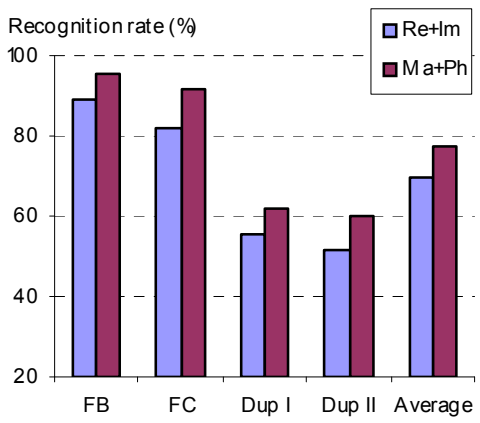

(c) 32 histogram bins

Figure 4. Comparative top-one recognition rates of $G_{-} L B P \mathrm{~s}$ with $11 \times 11$-sized subregions. 